\title{
ON THE LYAPOUNOV EXPONENTS OF SCHRÖDINGER OPERATORS ASSOCIATED WITH THE STANDARD MAP
}

\author{
J. BOURGAIN
}

\begin{abstract}
It is shown that Schrödinger operators defined from the standard map have positive (mean) Lyapounov exponents for almost all energies
\end{abstract}

\section{Definitions}

(1.1) Let $V$ be a bounded potential on $\mathbb{Z}_{+}$. Define $\omega(V)=\left\{W=\left(W_{n}\right)_{n \in \mathbb{Z}} ;\right.$ there is a sequence $n_{j} \rightarrow \infty$ such that $\left.d\left(S^{n_{j}} V, W\right) \rightarrow 0\right\}$ where

$d\left(V, V^{\prime}\right)=\sum 2^{-n}\left|V_{n}-V_{n}^{\prime}\right|$

and

$\left(S^{k} V\right)(n)=V(n+k)$.

(1.2) Let $A \subset \mathbb{R}$, mes $(A)>0$. Denote

$\mathcal{R}(A)=\left\{\right.$ bounded potentials $W=\left(W_{n}\right)_{n \in \mathbb{Z}}$ that are reflectionless on $\left.A\right\}$

and $\mathcal{R}^{C}(A)=\left\{W \in \mathbb{R}(A) ; \sup _{n}\left|W_{n}\right| \leq C\right\}$

$W \rightarrow H=W+\Delta$ and let $G(z)$ be the Green's function of $H$.

Recall that $W$ is reflectionless on $A$ if

$$
\operatorname{Re} G(t)(n)=0 \text { for a.e. } t \in A \text { and every } n \in \mathbb{Z} \text {. }
$$

2. Remling Theorems (see $[R]$ ).

(2.1) $\omega(V) \subset \mathcal{R}\left(\sum_{a c}(V)\right) \quad([\mathrm{R}]$ Theorem 1.4)

Typeset by $\mathcal{A} \mathcal{M S}-\mathrm{T}_{\mathrm{E}} \mathrm{X}$ 
(2.2) $R^{C}(A)$ is compact

([R] Proposition 4.1, (d))

(2.3) The restriction maps $\mathcal{R}(A) \rightarrow \mathcal{R}_{ \pm}(A)$ are injective ( \pm refers to $\pi_{\mathbb{Z}_{ \pm}}$).

and for any constant $C$, the map

$$
\mathcal{R}^{C}(A) \rightarrow \mathcal{R}_{+}^{C}(A) \text { is uniformly continuous }
$$

([R] Proposition 4.1 (c), (e))

(2.4) $W \in \mathcal{R}(A) \Rightarrow A \subset \sum_{a c}\left(W_{ \pm}\right)$

\section{Lyapounov Exponents}

Let $T$ be a measure preserving homeomorphism of a compact metric space $\Omega$ endowed with a probability measure $\mu$ that changes any non-empty open subset (we do not assume $T$ ergodic).

Let $\varphi \in \mathcal{C}(\Omega)$.

Denote half-line $S O$

$$
H_{x}=\varphi\left(T^{n} x\right) \delta_{n}+\Delta
$$

and

$$
\begin{aligned}
& M_{N}(E, x)=\prod_{N}^{1}\left(\begin{array}{cc}
E-\varphi\left(T^{n} x\right) & -1 \\
1 & 0
\end{array}\right) \\
& L_{N}(E)=\frac{1}{N} \int \log \left\|M_{N}(E, x)\right\| \mu(d x)
\end{aligned}
$$

Let $A \subset \mathbb{R},|A|>0$ and assume

$$
\varliminf_{N} L_{N}(E)=0 \text { on } A .
$$

Then

$$
A \subset \sum_{a . c}\left(V_{x}\right) \text { for } \mu \text { a.e.x }
$$

\section{Proof.}

Let

$$
\mu=\int \beta \alpha(d \beta)
$$

be the ergodic decomposition of $\mu$.

By Fubini, for $E \in A$

$$
L_{N}(E)=\int\left\{\frac{1}{N} \int \underset{2}{\log \left\|M_{N}(E, x)\right\| \beta(d x)}\right\} \alpha(d \beta)
$$


and

$$
\int\left\{\frac{\lim }{N}\left(\frac{1}{N} \int \log \left\|M_{N}(E, x)\right\| \beta(d x)\right)\right\} \alpha(d \beta) .
$$

Since $\beta$ is ergodic, it follows that for $\alpha$ - a.e. $\beta$

$$
\frac{1}{N} \log \left\|M_{N}(E, x)\right\| \rightarrow 0 \text { for } \beta \text { - a.e. } x \in \Omega
$$

Again by Fubini, (3.3) holds for a.e. $E \in A$ and $\beta$ - a.e. $x \in \Omega$

By Kotani theory, this implies that

$$
A \subset \sum_{a c}\left(H_{x}\right) \text { for } \beta-\text { a.e. } x \in \Omega \text {. }
$$

Since (3.4) is valid for $\alpha$ - a.e. $\beta,(3.2)$ follows.

\section{Use of recurrence}

Let $T$ be as in $\S 3$.

Let $V_{x}=\left(\varphi\left(T^{n} x\right)\right)_{n \in \mathbb{Z}_{+}}$

Then

$$
V_{x} \in \pi_{\mathbb{Z}_{+}}\left(\omega\left(V_{x}\right)\right) \text { for } \mu \text { - a.e. } x \in \Omega \text {. }
$$

\section{Proof.}

By Poincaré recurrence lemma, for $\mu$ - a.e. $x \in \Omega$, there is a sequence $n_{j} \rightarrow \infty$ such that

$$
T^{n_{j}} x \rightarrow x
$$

Hence

$$
\varphi\left(T^{n_{j}+k} x\right) \rightarrow \varphi\left(T^{k} x\right) \equiv W_{x}(k)
$$

for all $k \in \mathbb{Z}$ and

$$
d\left(W_{x}, V_{T^{n_{j}} x}\right) \rightarrow 0
$$

It follows that

$$
W_{x} \in \omega\left(V_{x}\right)
$$

and obviously $V_{x}=\pi_{\mathbb{Z}_{+}}\left(W_{x}\right)$.

By (2.1), (4.1) implies

$$
V_{x} \in \mathcal{R}_{+}\left(\sum_{a c}\left(V_{x}\right)\right) \text { for } \mu \text { a.e } x \text {. }
$$


From (4.3), (3.2)

$$
V_{x} \in \mathcal{R}_{+}(A) \text { for } \mu \text { a.e. } x
$$

thus $V_{x} \in \mathcal{R}_{+}^{C}(A)$ for $\mu$ a.e. $x$ and since $\mathcal{R}_{+}^{C}(A)$ is compact by (2.2) and $V_{x}$ depends continuously on $x$, we conclude that

$$
V_{x} \in \mathcal{R}_{+}(A) \text { for all } x \in \Omega
$$

( $\Omega=$ closure of any subset of full measure).

Finally, by (2.4)

$$
A \subset \sum_{a c}\left(V_{x}\right) \text { for all } x \in \Omega \text {. }
$$

\section{Application to standard map}

Let $T=T_{\lambda}$ be the standard map on the torus $\mathbb{T}^{2}=\Omega$ with sufficiently large $\lambda$. Thus

$$
f_{\lambda}(x, y)=(-y+2 x+\lambda \sin 2 \pi x, x) .
$$

Let $\varphi \in \mathcal{C}^{1}\left(\mathbb{T}^{2}\right)$ be a fixed not-constant function.

If the corresponding $\mathrm{SO}$ has vanishing Lyapounov exponents for $E \in A,(4.6)$ implies

$$
A \subset \sum_{a c}\left(\left\{\varphi\left(T^{n} x\right)\right\}_{n \in \mathbb{Z}_{+}}\right.
$$

for all $x \in \Omega$.

By Duarte's work (theorem A in $[\mathrm{D}]$ ), there is an invariant hyperbolic set $\Lambda=\Lambda_{\lambda} \subset \Omega$ such that $\left.T\right|_{\Lambda}$ is conjugate to a Bernoulli shift. In particular for $x \in \Lambda,(5.2)=\phi$. Furthermore, Duarte's result asserts that each point in $\mathbb{T}^{2}$ is within a $4 \lambda^{-\frac{1}{3}}$-neighborhood of $\Lambda$, so that $\varphi$ will not be constant on $\Lambda$ for $\lambda$ large enough. Hence (5.2) restricted to $\Lambda$ is non-deterministic and Kotani's theorem implies that $A$ is of zero-measure (contradiction).

Hence we proved

Proposition. For $\lambda>\lambda_{0}$, the $S O$ (5.2) associated to the standard map $T_{\lambda}$ has positive (mean) Lyapounov exponents for a.e. $E \in \mathbb{R}$.

Acknowledgement: The author is grateful to A. Avila, S. Sodin and T. Spencer for some discussions on this topic.

\section{REFERENCES}

[D]. P. Duarte, Plenty of elliptic islands for the standard family of area preserving maps, Ann.Inst. H. Poincaré Anal. Non linéaire 11 (1994), no 4, 359-409.

[R]. C. Remling, The absolutely continuous spectrum of Jacobi matrices, arXiv:1007.5033. 